ISBN 978-93-84468-80-4

International Conference on Recent Trends in Engineering and Technology

(RTET-2016))

Pattaya (Thailand) Dec. 14-16, 2016

\title{
The Residual Vibration Control of Robot Arm with Length- Changing Using Input Shaping Filter
}

\author{
Un-Hwan Park ${ }^{1}$, Jun-Hyeok Heo ${ }^{1}$, Tae-Hyeon $\mathrm{Oh}^{1}$, In-Sung Lee ${ }^{1}$, and Dae-Gyu Park ${ }^{1}$ \\ ${ }^{1}$ KOTMI (Korea Textile Machinery Institute)
}

\begin{abstract}
This paper shows that we reduced the residual vibration of the robot arm with length-changing using input shaping. We developed the two-way transfer robot for the 5th generation glass. We applied a hump input shaping filter to robot system to control the residual vibration of the robot. While robot transfers the glass, the natural frequency of robot arm is changed. Even though it is changed, input shaping filter with a hump has also good effect for this system
\end{abstract}

Keywords: Input shaping filter, residual vibration, 2-way transfer robot, natural frequency, vibration control

\section{Introduction}

In industrial fields that use the cranes or cargo carrying robot with a flexible structure, the residual vibration should be controlled for the precise position control. A lot of technique have been used to control the residual vibration. Specially, input shaping technique has been much used for the residual vibration of flexible structures. Input shaper is composed of multiple impulses. Input signal is formed to the signal that the residual vibration doesn't occur by a convolution with input shaping filter. Input shaping technique based on dividing the two-step input was introduced as posicast techniques [1]. The first impulse is applied to the system and later half period of oscillation the second impulse applied. the oscillation due to the first impulse is opposite in phase to it by the second impulse later half period of oscillation. Therefore, oscillation by first impulse is cancelled by second impulse. Input shaping filter is designed with the information of the frequency and the damping ratio of the system. However, if the characteristics of the system is changed, its performance is tends to be remarkably lower. It has been conducted much research on the robustness of the input shaping technique. By adding constraints, Singer and Seering developed input shaping to 3 impulses and obtained a good performance by applying to the robot system [2]. Singhose presented a multi-hump EI input shaper permitted residual vibration to some extent(for example 5\%) and has broad frequency range for residual vibration reduction [3-5]. When the robot with a flexible beam picks up the things, both of the frequency and the damping are changed at the same time. Park presented a robust multi-hump Convolution (CV) shaping filter for simultaneously changing of both the frequency and damping [6].

This paper shows that we reduce the residual vibration of the robot arm with length-changing using input shaping. We developed the two-way transfer robot for the 5th generation glass. We applied a hump input shaping filter to robot system to control the residual vibration of the robot. While robot transfer the glass, the frequency is changed. Even though it is changed, input shaping filter with a hump has also good effect for this system. 


\section{The Design of Input Shaping Filter}

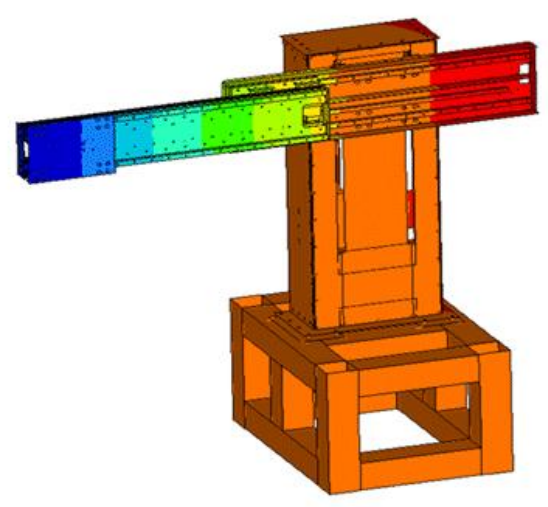

Fig 1 The transfer robot system

The developed robot has a cantilever structure capable of two-way transfer as shown in Fig.1. The vibration caused by loading is generated in a vertical direction during the transfer of the glass. This residual vibration is controlled using the input shaping techniques. because the length of the robot arm is changed during transfer, the natural frequency of the robot is changes. We use a humped input shaping filter with robust for frequency changing. it is permitted 5\% residual vibration and has broad frequency range for residual vibration reduction. When we design input shaping filter, the measured frequency was used. We took the average of the frequencies when the robot arm was extended to end and to the middle. The frequency is $18.2 \mathrm{~Hz}$. We actually measured the frequency of the robot arm using impact hammer as shown in Fig2.

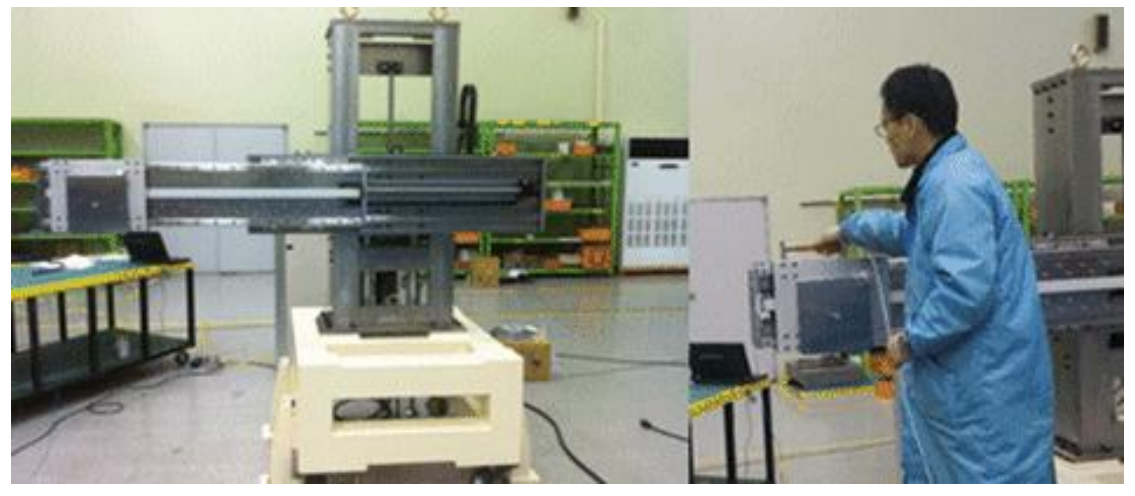

Fig. 2 The measurement of natural frequency for robot arm

The humped input shaping filter was designed in the z-domain. Fig. 3 shows the location of zeros in the zdomain. If two zeros is located in the both sides of the system pole, there is a hump in the sensitivity curve as shown in Fig. 4. The amplitude of hump is different as the distance of pole and zero. If it is far, the amplitude is big. So, we decided 5\% hump. Input shaping filter equation shows in (1). 


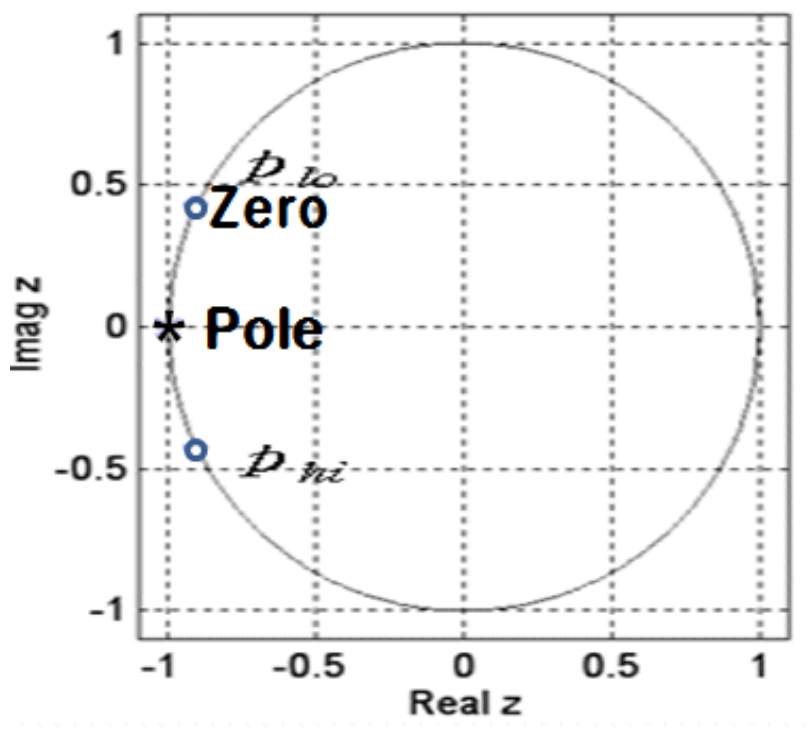

Fig. 3 The location of zeros in the z-domain

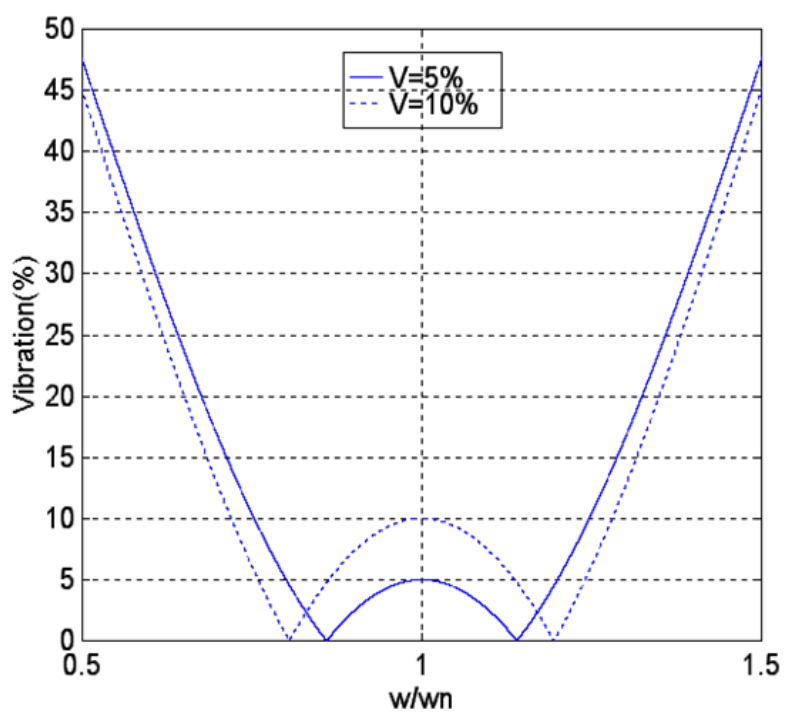

Fig. 4 The sensitivity of input shaping filter with a hump

$$
\begin{aligned}
H(z) & =\frac{1}{K}\left(1-p_{l o} z^{-1}\right)\left(1-p_{h i} z^{-1}\right) \\
& =\frac{1}{K}\left(1-\left(p_{l o}+p_{h i}\right) z^{-1}+p_{l o} p_{h i} z^{-2}\right)
\end{aligned}
$$

here, $\mathrm{K}$ : the sum of the coefficient for $\mathrm{z}^{0}, \mathrm{z}^{-1}, \mathrm{z}^{-2}$

$$
p_{\text {hine }}=e^{j\left(1 \pm \frac{\Delta_{\alpha}}{\omega_{n}}\right)=}
$$


The amplitude of input shaping filter should be normalizec $\underline{\Delta \omega}$ to change the amplitude of input signal. That is the reason why it is divided by $\mathrm{K}$. In order to has $5 \%$ hump, $\omega_{n}$ is 0.14 . Finally, the equation of input shaping filter is shown in (2).

$$
H(z)=A_{0}+A_{1} z^{-1}+A_{2} z^{-1}
$$

here, $\mathrm{A}_{0}=0.2676, \mathrm{~A}_{1}=0.4648, \mathrm{~A}_{2}=0.2676$

It means that impulse A0, A1 and A2 applied to the robot system at intervals of half period for the robot arm's oscillation as shown in Fig. 5. T is the half period for the robot arm's oscillation.

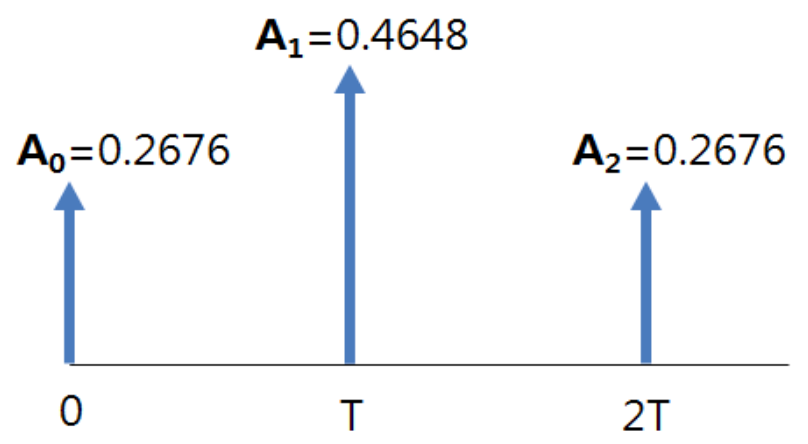

Fig. 5 The amplitude of input shaping filter with a 5\% hump

\section{The Experiment for Robot Arm}

If input acceleration profile is convoluted with input shaping filter(impulse signal), the input signal that residual vibration isn't generated is made as shown in Fig. 6 . The residual vibration was average $3 \mathrm{~m} / \mathrm{s}^{2}$ when robot arm moved to the end without input shaping filter. But when input shaping filter was applied to robot system, it was reduced to around $1 \mathrm{~m} / \mathrm{s}^{2}$ as shown in Fig. 7 . We confirmed that more than $66 \%$ of the residual vibration was reduced.

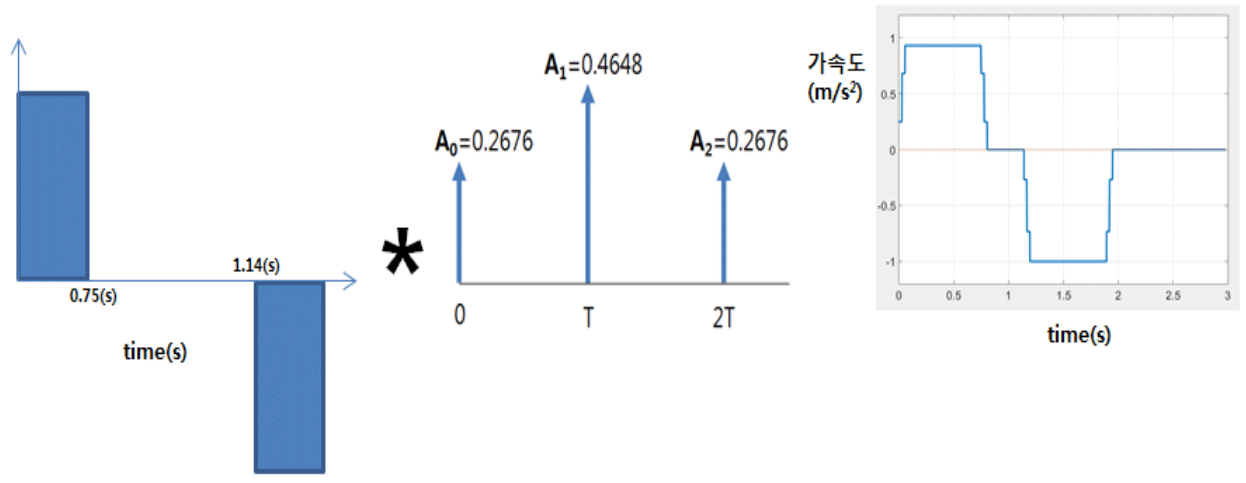

Fig. 6 The convoluted signal with input acceleration signal and impulses 


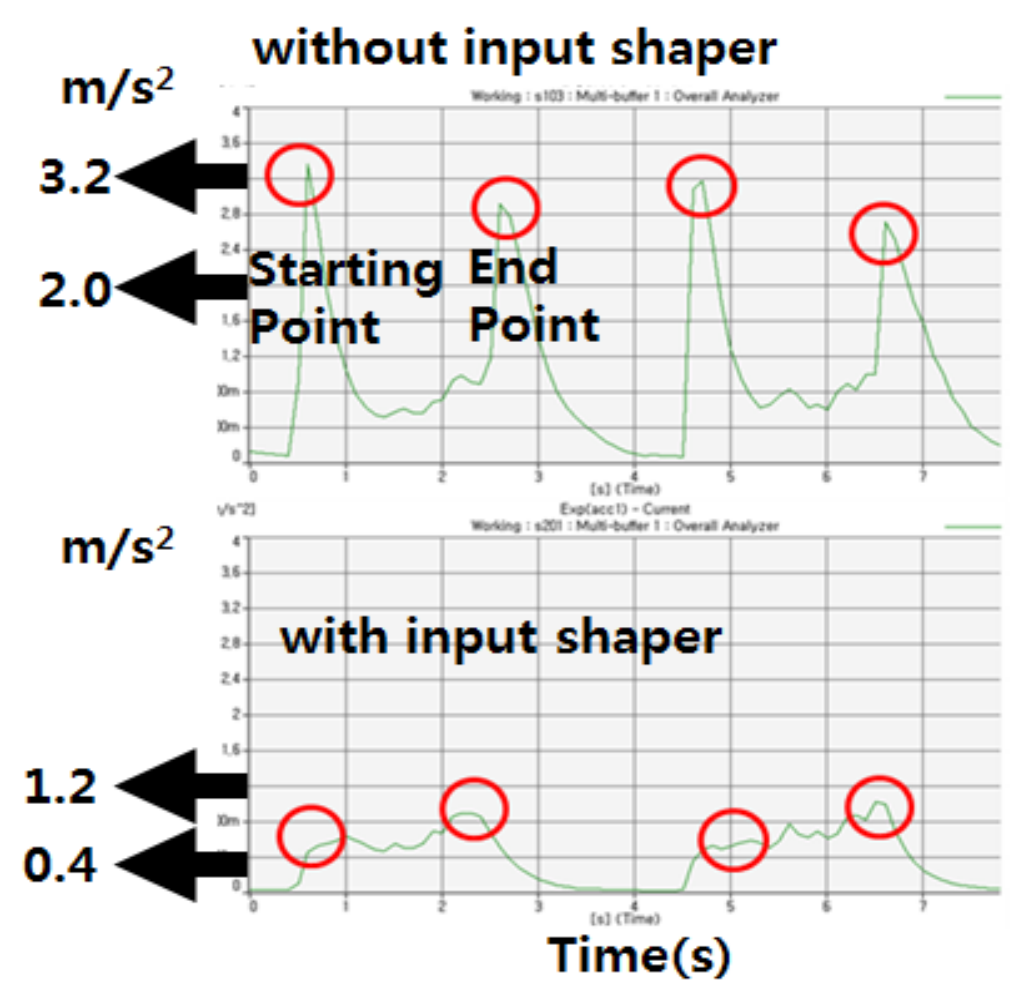

Fig. 7 The experiment result for the residual vibration control of robot arm.

\section{Conclusion}

Input shaping filter was applied to the residual vibration control of cantilever glass transfer robot. It was confirmed that the residual vibration was reduced by more than $66 \%$ at the test result. The frequency is changed as the length of the cantilever. In this paper, it shows that input shaping filter is effective for the system of variable frequency.

\section{Acknowledgements}

This research was supported by the Ministry of Trade, Industry \& Energy(MOTIE) , Korea Institute for Advancement of Technology(KIAT) through the Development Program for Robot Industry Cluster.

\section{References}

[1] Tallman, Smith, "Analog Study of Dead-Beat Posicast Control," IRE Transactios on Automatic Control, Vol. AC-3, pp14-23, 1958.

https://doi.org/10.1109/TAC.1958.1104844

[2] N.C. Singer, W.P. Seering, "Preshaping Command Inputs to Reduce System Vibration," Transactions of the ASME, Journal of Dynamic Systems, Measurement and Control, Vol. 112, pp76-82, 1990.

https://doi.org/10.1115/1.2894142

[3] W.E. Singhose, N.C. Singer, "Extra-Insensitivity Input Shapers for Controlling Flexible Spacecraft," Guidance, Control, and Dynamics Vol. 19. No. 2, 1996, March-April.

[4] L.J. Porter, W.E Singhose, T.D. Tuttle, N.C. Singer, "Vibration Reduction Using Multi-Hump Input Shapers," Transactions of the ASME, J DSMC Vol. 119, pp320-326, 1997.

[5] W.E. Singhose, W.P. Seering, N.C. Singer, "Time-Optimal Negative Input Shapers," ASME Vol. 119, pp198-205, 1997.

[6] U. H. Park, J. W. Lee, "Design of Robust Multi-Hump CV Shaper For Variation of Frequency and Damping Ratio" The Journal of KSME Vol. 26-1 pp.67-73 2002.1 